\title{
INDUCING PINNING STATES AND LOCALIZED PATTERNS IN A MONOLAYER OF LATERALLY ATTRACTING ADATOMS*
}

\author{
Sergio E. Mangioni ${ }^{\dagger}$, Roberto R. Deza \\ Mar del Plata Institute for Physics Research (IFIMAR) $)^{\ddagger}$ \\ Faculty of Exact and Natural Sciences (FCEyN) \\ National University of Mar del Plata (UNMdP), \\ Deán Funes 3350, B7602AYL Mar del Plata, Argentina
}

(Received April 24, 2017; accepted June 13, 2017)

The so-called "entropic mechanism" for ordering noise-induced phase transitions has been formerly used to stabilize nanopatterns in a realistic model for a monolayer of laterally interacting adsorbates. Here, we use this mechanism to stabilize pinning states and induce stochastic localized patterns ("dissipative solitons") on the monolayer. The key to achieve that goal is to displace only one of the stable homogeneous states toward field values at which the lateral interactions can elicit stable nanopatterns. At larger noise intensities, the "soliton" locations fluctuate with larger amplitude. However, since more localized patterns are elicited by the noise, their motion becomes confined when looked at longer timescales.

DOI:10.5506/APhysPolB.48.849

\section{Introduction}

Recently, research on localized patterns - bubbles of metastable homogeneous states ${ }^{1}$ — has gained momentum [3-6]. Found long ago in morphogenesis [7-10], they have been since observed in a wide range of situations of technological interest ${ }^{2}$ : magnetic materials, gas discharge systems,

* Presented by R. Deza at the $28^{\text {th }}$ Marian Smoluchowski Symposium, Kraków, Poland, September 14-17, 2015.

$\dagger$ Member of CONICET, Argentina.

¥ CONICET-UNMdP.

${ }^{1}$ As far as their interaction is not the issue, localized patterns are also referred to in the literature as "dissipative solitons" (see e.g. [1, 2]). Although strictly an abuse of language, the word "soliton" is hereafter employed only as a shorthand for "localized pattern".

2 A list of references (to the date of their publication) can be found in [11, 12]. 
optics and liquid crystals, mechanical systems (as e.g. parametrically driven systems and granular media) and chemical systems, with special mention of heterogeneous catalysis. Their present interest (as well as that of closely related chimera states) is being spurred by nanotechnology and optical fiber industries.

Almost from the outset, it was clear that bistable scalar reaction-diffusion (RD) media in 1D were not able to sustain stable localized patterns [13]. An interesting view of this instability is to interpret the solvability condition (determining the time derivative of the "soliton" width) as an attracting force between the kinks bounding the bubble, which makes it shrink and disappear [11, 12]. In a recent article [14], we have shown that a simple frontenabling bistable scalar 1D RD medium (in fact, a pitchfork normal form) does sustain localized patterns, provided that the two following ingredients are added:

- a weak aggregating current $J_{\mathrm{A}}(x, t)$, which can in certain situations (e.g. when due to a very short-range attracting potential) be assimilated to an "antidiffusive" term [15, 16], and

- a multiplicative (state-dependent) noise, of the kind leading to entropic noise-induced phase transition [17, 18].

$J_{\mathrm{A}}(x, t)$ gives rise to periodic patterns, but for the field values corresponding to the homogeneous states (HS), these are "washed out" by diffusion. A successful strategy to stabilize the periodic patterns has been to enlarge the system with a further irreversible reaction [19].

A different strategy - recently proved to be successful [20-22] - is to submit the medium to an external spatiotemporal Gaussian white noise, with a state-dependent coefficient. This will modify the drift or "reaction term" and lead to new stable field values. So if the state-dependent coefficient is suitably tailored, the periodic patterns raised by the weak aggregating current will survive the effect of diffusion for the new stable field values.

Now, the noise term can be made to perturb only one HS. In such a case, the new bistability arising between the pattern and the remaining HS will lead to pinning states in the presence of fronts, and localized patterns will arise through this pinning mechanism [14]. The purpose of this article is to show the working of this mechanism in a realistic model $[15,16]$ : a monolayer of laterally attracting adatoms.

Section 2 is devoted to a description of the model which will become our workbench; Section 3, to the strategy for inducing pinning states and localized patterns by means of a multiplicative noise; The remaining sections, to the numerical results and conclusions. 


\section{A monolayer of attracting adatoms}

Modern microscopy techniques such as STM and field ion ones, have revealed - in real time and with almost atomic resolution - the existence of nanometer sized patterns. This exciting discovery makes the system relevant for industrial applications such as electronic devices or nanosize heterogeneous catalysis.

Starting from an underlying microscopic master equation, a nonlocal mesoscopic kinetic equation was derived in the mid nineties that explicitly takes into account the attractive lateral interactions (ALI) between adsorbates at coverage $0 \leq \phi(x) \leq 1[15,16]$. As usual for RD models, the system was divided into cells whose local dynamics ("reaction" term) represents adsorption and (ALI-affected) desorption. Transport between cells was represented by an effective "diffusion" coefficient $D_{\text {eff }}(\phi)$ which, in addition to the real diffusion process, accounts for the ALI-driven current flow. This procedure makes sense whenever the cells can be regarded as "macroscopic" with respect to local dynamics, so their length $l$ must be large enough. On the other hand - in order to describe pattern formation - $l$ must be much smaller than the typical lengthscale of the inhomogeneities. In the present case, this is given by the ALI midrange $r_{\mathrm{a}}$ and is nanometer sized, which renders the condition $l \ll r_{\mathrm{a}}$ very restrictive.

Neglecting parameter fluctuations, the mesoscopic equation describing the dynamics is thus assumed to be an RD equation with non-Fickian diffusion. At variance with true diffusion processes, which tend to eliminate inhomogeneities, the aggregating drive of ALI tends to generate them [20-28]. However, since the ALI also affect the desorption process, they cannot by themselves sustain those inhomogeneities in time, and the HS turn out to be stable. Periodic patterns can be stabilized with the addition of an irreversible chemical reaction [19]. Recently, we succeeded to do so by means of a suitable multiplicative noise [20-22]. Much in the same way by effect of adsorption, desorption, diffusion, and ALI-driven currents localized patterns with sizes ranging from sub-micrometer to nanometer can also arise in metal surfaces, but only with resort to an additional stabilizing mechanism. With the addition of a simple irreversible chemical reaction for instance, stable localized structures may spontaneously develop through a nonequilibrium self-organization process [29].

Now, although ALI-dependent desorption dynamics does not by itself lead to stable localized patterns ${ }^{3}$, it enables bistability within the parameter region in which $D_{\text {eff }}(\phi)<0$ (what we call hereafter "the constructive

${ }^{3}$ For ALI-independent desorption (a questionable hypothesis [30, 31]) stable localized patterns have been reported in the absence of an additional stabilizing chemical reaction [32]. 
region"). Depending on the adsorption and desorption coefficients, two HS (here denoted $\phi_{\mathrm{d}}$ and $\phi_{\mathrm{u}}$, to indicate respectively lower and higher coverage) are obtained. Both are stable under either homogeneous or inhomogeneous perturbations.

Although localized structures have not been reported so far in this system, it has been shown [20-22] that when a particular type of multiplicative noise is incorporated to the dynamics, this can push one HS (either $\phi_{\mathrm{d}}$ or $\phi_{\mathrm{u}}$ ) toward $\phi$ values in the "constructive region", where it can be destabilized by an inhomogeneous perturbation, giving rise to spatially periodic patterns and/or bistable (HS-pattern) situations (the detailed mechanism is illustrated in [33, 34]).

The normalized dynamics of the coverage $\phi(x)$ by a monolayer of adsorbates undergoing ALI can be described by [16]

$$
\partial_{t} \phi=Q(\phi)-\partial_{x} J_{\mathrm{A}}+\partial_{x x} \phi
$$

where

$$
Q(\phi)=\alpha(1-\phi)-\phi e^{U^{[\phi]}}
$$

accounts for adsorption - on void sites, hence the factor $1-\phi$ - and ALIsuppressed (through $e^{U^{[\phi]}}$ ) desorption ( $\alpha$ is the vapor pressure times the rate-constant ratio), $\partial_{x x} \phi$ for "bona fide" diffusion, and

$$
J_{\mathrm{A}}(x)=\varepsilon_{0} \phi(1-\phi) \partial_{x} U^{[\phi]}
$$

is the ALI-driven current. Here:

- $U^{[\phi]}(x)=-\int \mathrm{d} x^{\prime} f\left(x^{\prime}-x\right) \phi\left(x^{\prime}\right)$, a functional of $\phi(x)$, is the average ALI effect,

- $f(x)$ the ALI potential (whose detailed form is irrelevant as far as it has midrange $r_{\mathrm{a}}$ ),

- $\varepsilon_{0}$ a measure of the ALI strength (relative to the adsorbate's mean kinetic energy),

- $\phi(1-\phi)$ limits the adsorbate motion to free places.

By defining an effective diffusion coefficient

$$
D_{\text {eff }}(\phi)=1-\varepsilon_{0} \phi(1-\phi) \frac{\partial_{x} U^{[\phi]}}{\partial_{x} \phi},
$$

where the smoothly varying ratio $\partial_{x} U^{[\phi]} / \partial_{x} \phi$ is to be regarded as a mere coefficient, Eq. (1) reads

$$
\partial_{t} \phi=Q(\phi)+\partial_{x}\left[D_{\text {eff }}(\phi) \partial_{x} \phi\right]
$$


The solutions to Eq. (4) - and thus, to Eq. (1) - are stable HS and (only in the bistable regime) fronts propagating towards one or the other HS ( $\phi_{\mathrm{d}}$ or $\phi_{\mathrm{u}}$, depending on which side of the ALI-modified point of the Maxwell construction the system is situated in).

Any new effect that causes the displacement of the HS toward field values so that $D_{\text {eff }}(\phi)<0$ will make it unstable, thus promoting the formation and stabilization of patterns [19-22]. That is why we call this regime the "constructive region".

\section{The "pushing" multiplicative noise}

The strategy to push one HS towards the "constructive region" relies on applying to the medium a space- and field-dependent Gaussian white noise $\eta(x, t)$ with zero mean and correlation

$$
\left\langle\eta(x, t) \eta\left(x^{\prime}, t^{\prime}\right)\right\rangle=\sigma^{2} \delta\left(x-x^{\prime}\right) \delta\left(t-t^{\prime}\right),
$$

and then tailor out its multiplicative factor $\sqrt{\Gamma(\phi)}$ for the task.

By introducing this multiplicative noise in equation Eq. (4), the dynamics are now governed by

$$
\partial_{t} \phi=Q(\phi)+\partial_{x}\left[D_{\mathrm{eff}}(\phi) \partial_{x} \phi\right]+\sqrt{\Gamma(\phi)} \eta(x, t) .
$$

In order to map the system into a non-gradient relaxational one, we rewrite equation (5) as

$$
\partial_{t} \phi=-\Gamma(\phi) \frac{Q(\phi)+\partial_{x}\left[D_{\mathrm{eff}}(\phi) \partial_{x} \phi\right]}{-\Gamma(\phi)}+\sqrt{\Gamma(\phi)} \eta(x, t) .
$$

Subsequently, we define a free energy functional $\mathcal{F}[\phi(x)]$ such that

$$
\frac{\delta \mathcal{F}[\phi(x)]}{\delta \phi(x)}=\frac{Q(\phi)+\partial_{x}\left[D_{\mathrm{eff}}(\phi) \partial_{x} \phi\right]}{-\Gamma(\phi)},
$$

so Eq. (5) can be written as

$$
\partial_{t} \phi=-\Gamma(\phi) \frac{\delta \mathcal{F}[\phi]}{\delta \phi(x)}+\sqrt{\Gamma(\phi)} \eta(x, t) .
$$

In those conditions, the corresponding stationary probability density function (SPDF) $P(\phi)$ is of Boltzmann's type $[17,18]$

$$
P(\phi) \propto \exp \left\{-\frac{2 \mathcal{F}_{\mathrm{eff}}[\phi]}{\sigma^{2}}\right\},
$$


in terms of an effective free-energy functional

$$
\mathcal{F}_{\text {eff }}[\phi]=\mathcal{F}[\phi]+B \sigma^{2} \ln [\Gamma(\phi)],
$$

where coefficient $B=1$ in Stratonovich's interpretation, and 2 in Itô's one. Fortunately, the average effect of this noise can be studied without explicit knowledge of the functional $\mathcal{F}[\phi(x)][20-22,24]$. It suffices to replace $Q(\phi)$ in Eq. (1) or Eq. (4) by

$$
Q_{\lambda}(\phi)=Q(\phi)-\lambda \frac{\mathrm{d} \Gamma}{\mathrm{d} \phi}
$$

with $\lambda:=B \sigma^{2} L, L$ being the (dimensionless) length of the $x$-domain. In this way, the equation that describes the average dynamics is written as

$$
\partial_{t} \phi=Q_{\lambda}(\phi)+\partial_{x}\left[D_{\mathrm{eff}}(\phi) \partial_{x} \phi\right] .
$$

Although in the absence of noise, the solutions to Eq. (10) are fronts propagating towards one or the other HS $\left(\phi_{\mathrm{d}}\right.$ or $\phi_{\mathrm{u}}$, depending on which side of the ALI-modified point of the Maxwell construction the system is situated), a suitable multiplicative noise is able to push the solution toward the "constructive region" [33], resulting in the formation and stabilization of a pattern [20-22].

Considering that:

- our primordial objective is to displace only one of the HS, in order to create a bistable pattern-SH situation (the noise should not affect the other HS),

- previous analytical and numerical results show that the noise pushes the system in the sense in which $\Gamma(\phi)$ decreases [20-22, 24],

- more recent results indicate that the larger the (negative) slope of $\Gamma(\phi)$ is, the more effective is the stochastic force to push the system toward a situation in which noise effects are minimized [33, 34].

We propose

$$
\Gamma_{\mathrm{u}}(\phi):=\exp [b(\phi-1)]
$$

to affect $\phi_{\mathrm{u}}$, and

$$
\Gamma_{\mathrm{d}}(\phi):=\exp [-b \phi]
$$

to affect $\phi_{\mathrm{d}}($ we use $b=10)$. These noise factors take the affected HS near their respective stability thresholds, so this device is not expected to work for too large $\sigma^{2}$ values (the HS intended to be affected would cease to exist). Since (as aforementioned) we have stabilized pinning states and localized patterns by using these factors in a normal form, we now intend to use them in the model described in the previous section. 


\section{Numerical results}

Adopting the (dimensionless) value $r_{\mathrm{a}}=10^{-2}$ for the ALI midrange, we have chosen the spatial discretization $\delta x=2.5 \times 10^{-2} r_{\text {a }}$ (so that $\delta x \ll$ $\left.r_{\mathrm{a}} \ll L\right)$ and solved Eq. (8) by the Heun method. According to Eqs. (2)-(3), parameter $\alpha$ determines $Q(\phi)$ and $\varepsilon_{0}$ determines $D_{\text {eff }}(\phi)$. We have adopted the values $\varepsilon_{0}=13, \alpha=0.03$ in Figs. $1-9$, and $\varepsilon_{0}=14, \alpha=0.001$ in Figs. 10-14.

\subsection{Pinning states}

The first task to undertake is the numerical proof that pinning states can indeed be stabilized by means of a multiplicative noise, with either $\Gamma_{\mathrm{u}}(\phi)$ — defined by Eq. (11) - or $\Gamma_{\mathrm{d}}(\phi)$, defined by Eq. (12). Here, we illustrate that in fact using $\Gamma_{\mathrm{d}}$, meant to displace the HS $\phi_{\mathrm{d}}$ toward the "constructive region". In the normal-form case, there was a region in parameter space such that pinning states could be stabilized even in the absence of a "pusher noise" [14]. In this realistic adsorption-desorption model instead, the "pusher noise" is always required [20-22].

We note that $\Gamma_{\mathrm{d}}$ decreases as $\phi$ increases, and becomes vanishingly small around $\phi_{\mathrm{u}}$. So when $\phi \sim \phi_{\mathrm{d}}$ and $\Gamma_{\mathrm{d}}$ is large, the drift term $-\lambda \mathrm{d} \Gamma / \mathrm{d} \phi$ in Eq. (9) - of stochastic origin — opposes the deterministic one $Q(\phi)$ and pushes the system toward higher field values. Conversely for $\phi>\phi_{\mathrm{d}}$, the stochastic force goes quickly to zero. The stationary state is achieved when both terms are in balance. For $\sigma^{2}$ high enough, the noise-affected HS will lie inside the constructive region, and then a pattern will emerge. Thus, we expect to create a bistable pattern-SH situation, which provides a route to pinning states.

In the absence of noise, the HS are always located outside the instability region, regardless of the parameter values. In the bistable region (between those HS), front solutions propagate towards the less stable HS unless the Maxwell condition is exactly fulfilled. We now attempt to use a multiplicative noise with factor $\Gamma_{\mathrm{d}}$ to "push" one HS inside the constructive region.

Introducing as an initial condition a hyperbolic tangent-like profile, no pinning states were observed (only usual front solutions). We then introduced an initial modulated step-like profile, of the type proposed by Pomeau [35]. Frame (a) in Fig. 1 shows that for a low modulation amplitude, a hyperbolic tangent-like profile results. In frame (b) instead, the modulation amplitude is large enough to generate a pinning-front. This means that both types of solution are possible: front solutions propagating towards the less stable HS, and pinning-fronts. Moreover, we have observed that they can coexist. 

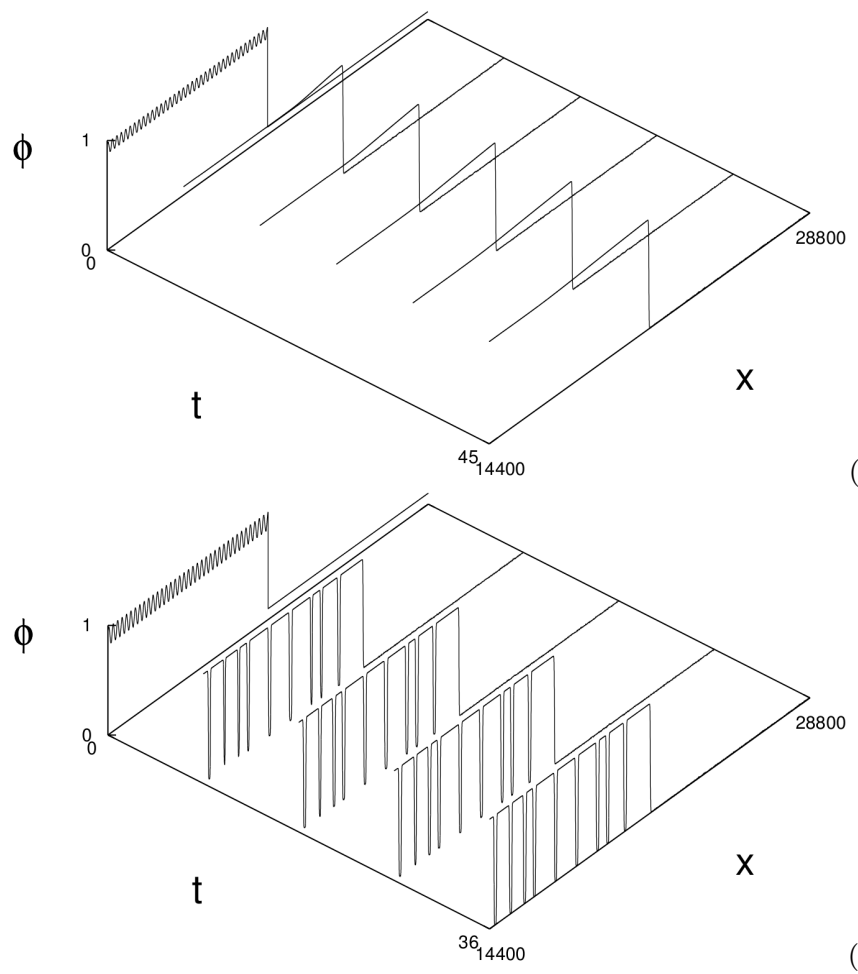

(a)

Fig. 1. Evolution of a modulated step-like profile of the type proposed by Pomeau [35], submitted to noise with $\sigma=2$ and $\Gamma_{\mathrm{d}}$, when the initial step amplitude is below (a) or above (b) the threshold to obtain pinning. Parameters: $\varepsilon_{0}=13, \alpha=0.03$.

\subsection{Localized patterns}

Now we undertake the second task - namely the stabilization of localized patterns by means of a multiplicative noise - always through solving Eq. (8) by the Heun method. In order to help this process (although as shown below, it turned out to be unnecessary in most cases), we introduced the initial condition

$$
\phi_{0}^{\mathrm{d}}(x):=\frac{1}{2}-f(x)
$$

when aiming to elicit " $S^{-}$solitons" (or "dips"), and

$$
\phi_{0}^{\mathrm{u}}(x):=-\frac{1}{2}+f(x)
$$

when aiming to elicit " $S^{+}$solitons" (or "bumps"). Here,

$$
f(x):=.48\left[\tanh \rho A\left(x-x_{f}+\delta\right)-\tanh \rho A\left(x-x_{f}-\delta\right)\right],
$$


where $\rho \gtrsim 1$, and parameter $\delta$ is half the "seeding" width. The "seeding" position $x_{f}$ was taken right at the center of the domain (namely $x_{f}=21,600 \delta x$ ), and the factor $A:=L_{\text {diff }} / r_{\mathrm{a}}$ is introduced because (whereas the equation is normalized with the diffusion length $\left.L_{\text {diff }}\right)$ the relevant parameter is $r_{\mathrm{a}}$.

\subsubsection{Numerical results: " $S^{-}$solitons" or "dips"}

As in the previous subsection, we adopt $\varepsilon_{0}=13, \alpha=0.03$. Figure 2 shows the noiseless evolution from the initial condition $\phi_{0}^{\mathrm{d}}(x)$ in Eq. (13). The fronts making up the "seeding" profile end up collapsing. The same occurs when noise with $\Gamma_{\mathrm{d}}$ is added, as far as $\sigma \lesssim 0.05$.

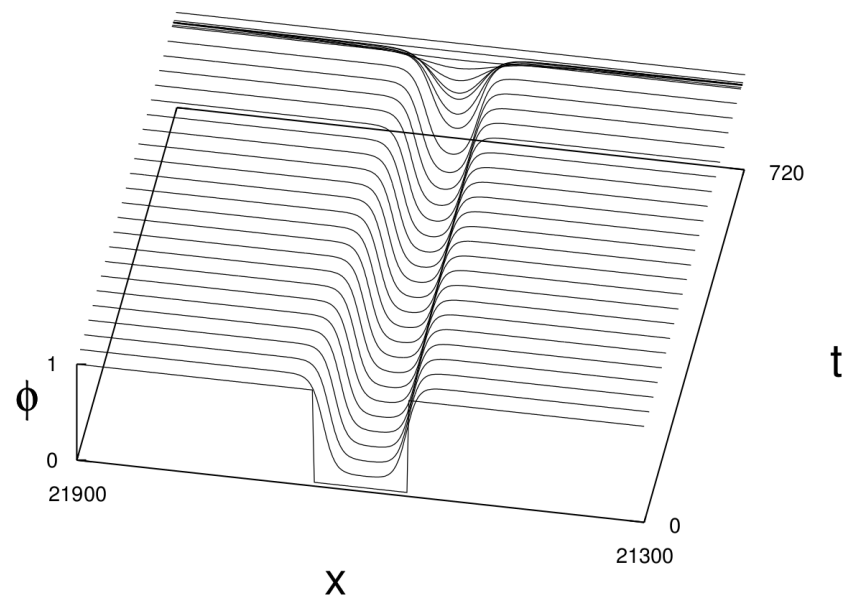

Fig. 2. Noiseless evolution from the initial condition $\phi_{0}^{\mathrm{d}}(x)$ in Eq. (13). Parameters: $\varepsilon_{0}=13, \alpha=0.03$.

Figure 3 shows the evolution from the same initial condition (and the same parameters) under noise with $\Gamma_{\mathrm{d}}$, for $\sigma=2$. An " $S^{-}$soliton" (a localized $\phi_{\mathrm{d}}$ region surrounded by $\phi_{\mathrm{u}}$ ) becomes stabilized by the multiplicative noise. Frame (b) - a zoom of frame (a) - shows that the "soliton" position fluctuates.

Figures 4 and 5 illustrate the fact that when the noise intensity $\sigma^{2}$ is further increased (in this case, $\sigma=15$ ), other localized patterns (besides the "seeded" one) emerge and stabilize spontaneously. Figure 4 shows the evolution of two " $S^{-}$solitons", one "seeded" and the other spontaneous. Figure 5 shows expanded views of this case, focusing respectively on the "seeded" and spontaneous localized patterns. These zooms also show that besides stabilizing the " $S^{-}$solitons", the noise also induces a stochastic wandering of their locations. By comparing Figs. 4 and 5 with Fig. 3 (corresponding to $\sigma=2$ ), we see that the wandering motion becomes restricted as $\sigma$ decreases. 


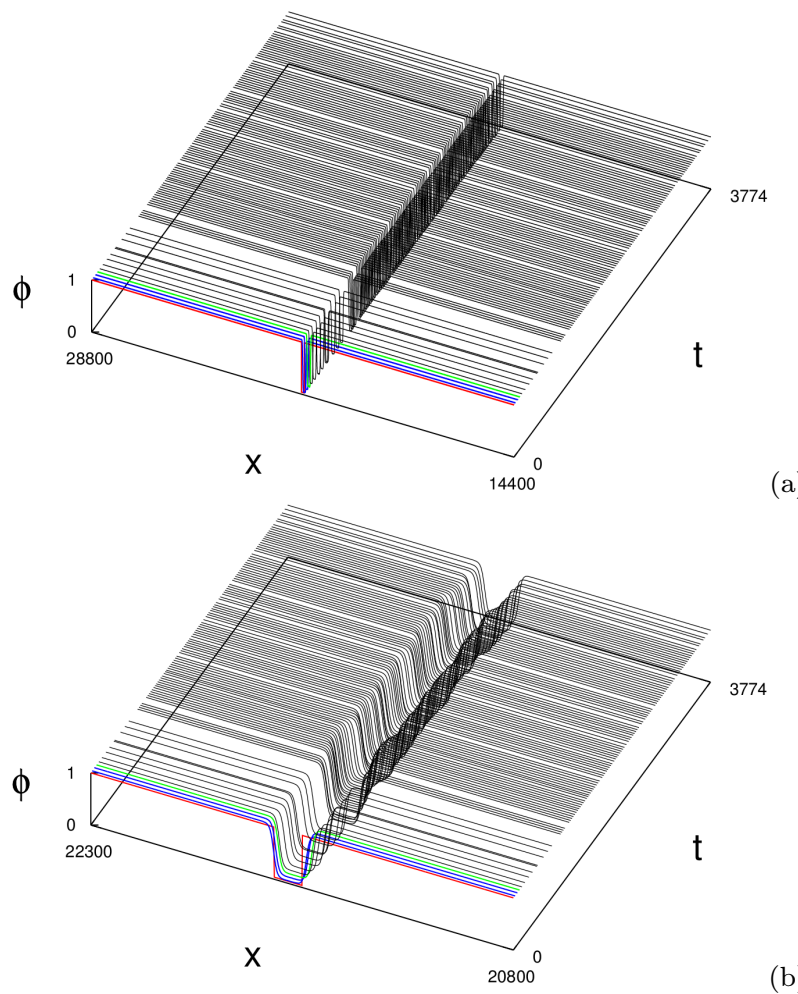

(a)

(b)

Fig. 3. (a) Evolution from the initial condition $\phi_{0}^{\mathrm{d}}(x)$ in Eq. (13), when the system is submitted to noise with $\Gamma_{\mathrm{d}}$ and $\sigma=2$. (b) A zoom of frame (a). Parameters: $\varepsilon_{0}=13, \alpha=0.03$.

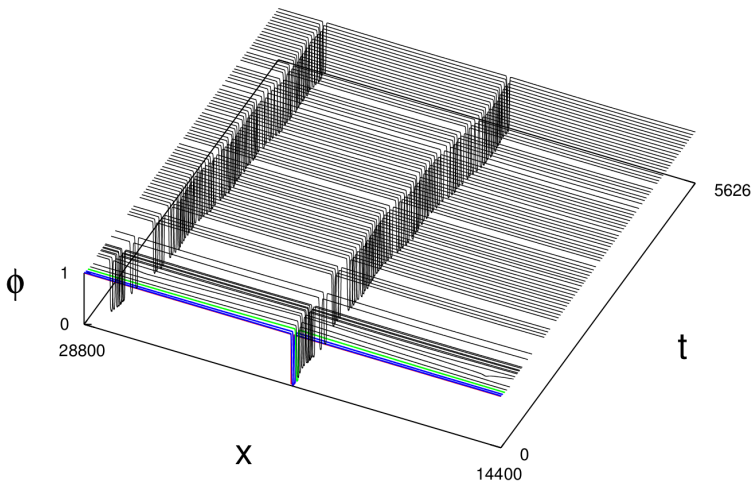

Fig. 4. Evolution of two localized patterns (one "seeded" and one spontaneous) from the initial condition $\phi_{0}^{\mathrm{d}}(x)$ in Eq. (13), when the system is submitted to noise with $\Gamma_{\mathrm{d}}$ and $\sigma=15$. Parameters: $\varepsilon_{0}=13, \alpha=0.03$. 


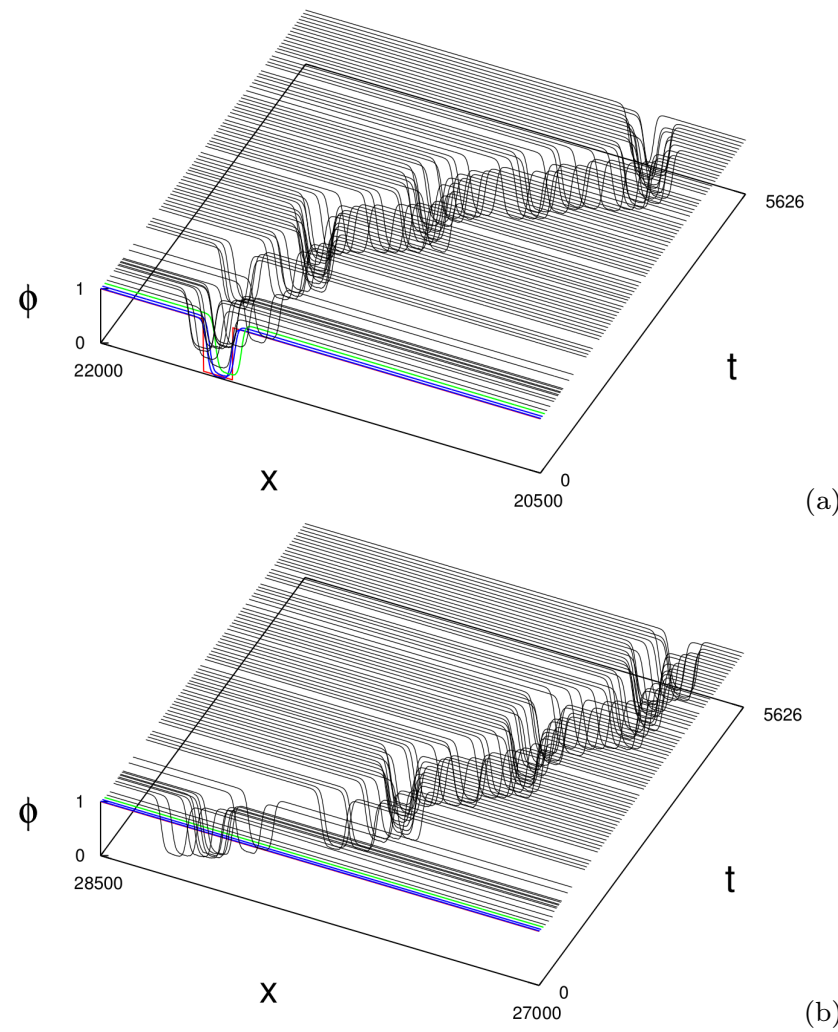

Fig. 5. (a) Zoom of the evolution of the "seeded" localized pattern in Fig. 4. (b) Zoom of the evolution of the spontaneous localized pattern.

Clearly, more spontaneous localized patterns emerge (and stabilize) and more stochastic becomes their motion as the noise intensity $\sigma^{2}$ keeps increasing. Figures 6 and 7 show the evolution of four " $S^{-}$solitons" (one "seeded" and three spontaneous) for $\sigma=20$. Figures 8 and 9 show the evolution of seven " $S^{-}$solitons" (one "seeded" and six spontaneous) for $\sigma=30$.

We note that the motion can be decomposed into a (stochastic but nevertheless somewhat well-defined) path, and shorter-timescale fluctuations around it. 


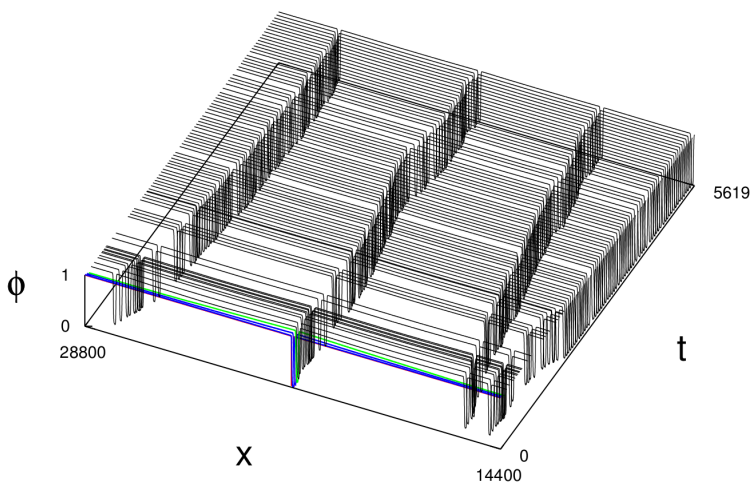

Fig. 6. Evolution of four localized patterns (one "seeded" and the other ones spontaneous) from the initial condition $\phi_{0}^{\mathrm{d}}(x)$ in Eq. (13), when the system is submitted to noise with $\Gamma_{\mathrm{d}}$ and $\sigma=20$. Parameters: $\varepsilon_{0}=13, \alpha=0.03$.
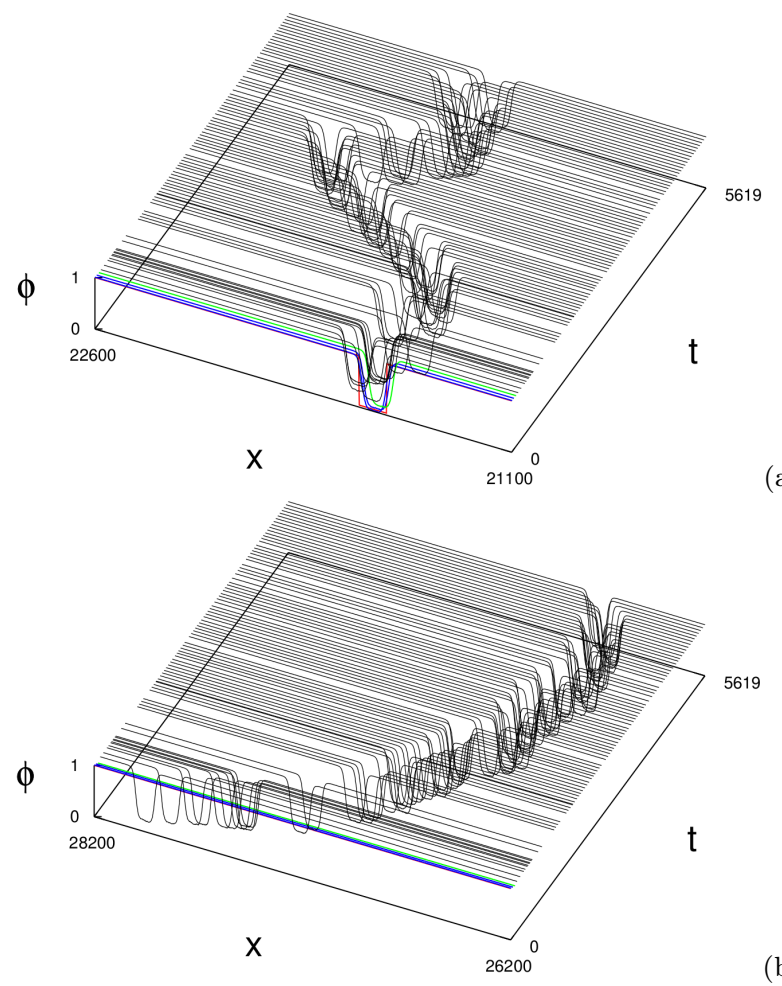

(a)

(b)

Fig. 7. (a) Zoom of the evolution of the "seeded" localized pattern in Fig. 6. (b) Zoom of the evolution of one of the spontaneous localized patterns. 


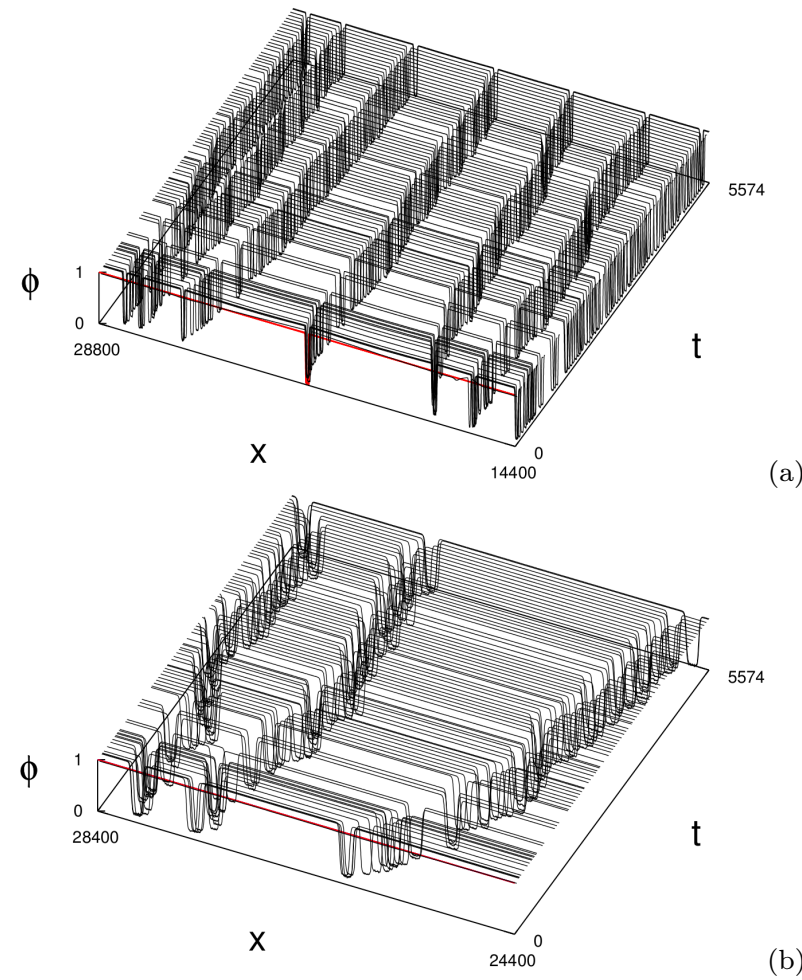

Fig. 8. Evolution from the initial condition $\phi_{0}^{\mathrm{d}}(x)$ in Eq. (13), when the system is submitted to noise with $\Gamma_{\mathrm{d}}$ and $\sigma=30$. (a) Evolution of seven localized patterns. (b) Zoom of frame (a), focalizing on the three spontaneous localized patterns at the left. Parameters: $\varepsilon_{0}=13, \alpha=0.03$.

By comparing Figs. 5, 7 and 9, we perceive that only the shorter-timescale stochastic motion becomes more intense as $\sigma$ increases, while the path becomes even better defined as $\sigma$ increases. Since also the number of localized patterns increases with $\sigma$, the above feature is likely to be due to the interaction between localized patterns. Although comparison of the two-"soliton" case of Figs. 4, 5 with the four-"soliton" one of Figs. 6, 7 is inconclusive with regard to the longer-timescale motion, comparison of the four-"soliton" case of Figs. 6, 7 with the seven-"soliton" one of Figs. 8, 9 shows that such motion appears to be more restricted. Then the closer together localized patterns are, the more restricted the longer-timescale motion becomes, whereas shorter-timescale motion always increases with $\sigma$. By turning again our attention to Figs. 6 and 8 (a), we observe that the localized patterns seem to repel each other when they are close enough. Moreover, the "seeded" localized pattern at the middle oscillates around its position, with a restricted motion. Probably, it is equally pushed from both sides. 


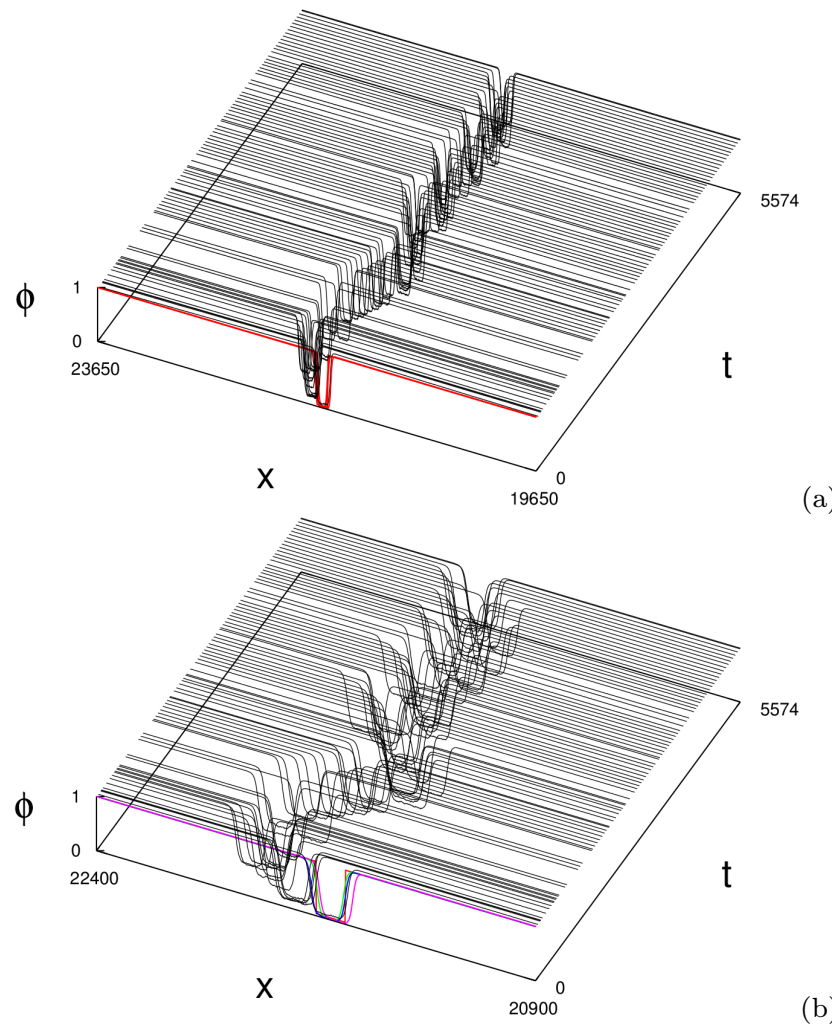

Fig. 9. (a) Zoom of the evolution of the "seeded" localized pattern in Fig. 8. (b) Zoom of the evolution of one of the spontaneous localized patterns.

On the other hand, if $\sigma$ is large enough ( $\sigma=35$ for instance), " $S^{-}$solitons" are destabilized. Hence, there is a window of noise intensity values where the localized patterns can be stabilized ( $\sigma$ between 0.05 and 35 for $\varepsilon_{0}=13$ and $\alpha=0.03)$.

\subsubsection{Numerical results: " $S^{+}$solitons" or "bumps"}

Throughout this subsection, we shall adopt $\varepsilon_{0}=14, \alpha=0.001$. In order to elicit " $S^{+}$solitons" (a localized $\phi_{\mathrm{u}}$ region surrounded by $\phi_{\mathrm{d}}$ ), we use $\Gamma_{\mathrm{u}}$. Figure 10 shows the evolution from initial condition $\phi_{0}^{\mathrm{u}}(x)$ in Eq. (14) under noise with $\Gamma_{\mathrm{u}}$, for $\sigma=10$. We explored a wide range of parameter values and found the same results whenever our criterion was satisfied. Figure 11 focuses on the evolution of a single " $S^{+}$soliton" for $\sigma=15$. A little enhancement of both motions (shorter- and longer-timescale ones) is observed. 


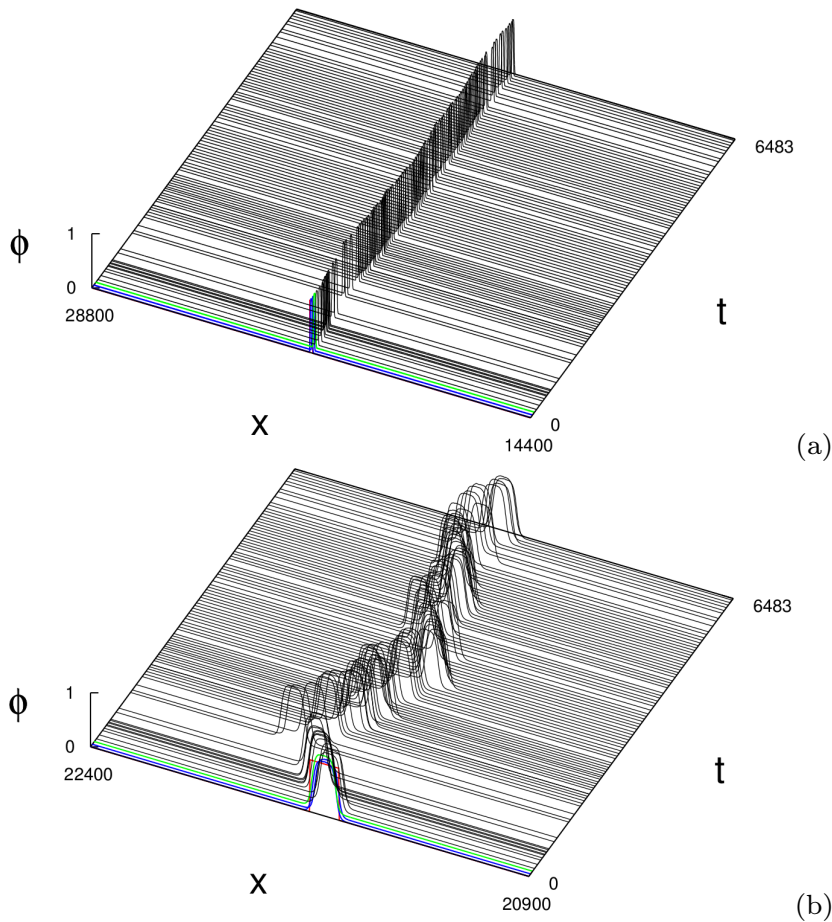

Fig. 10. (a) Evolution from the initial condition $\phi_{0}^{\mathrm{u}}(x)$ in Eq. (14), when the system is submitted to noise with $\Gamma_{\mathrm{u}}$ and $\sigma=10$. Only the "seeded" localized pattern is elicited. (b) Zoom of frame (a). Parameters: $\varepsilon_{0}=14, \alpha=0.001$.

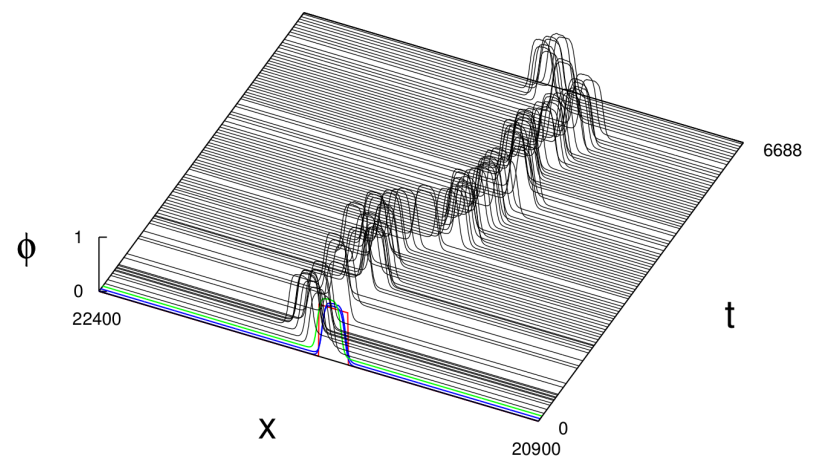

Fig. 11. Evolution from the initial condition $\phi_{0}^{\mathrm{u}}(x)$ in Eq. (14), when the system is submitted to noise with $\Gamma_{\mathrm{u}}$ and $\sigma=15$. Again, only the "seeded" localized pattern is elicited. Parameters: $\varepsilon_{0}=14, \alpha=0.001$. 


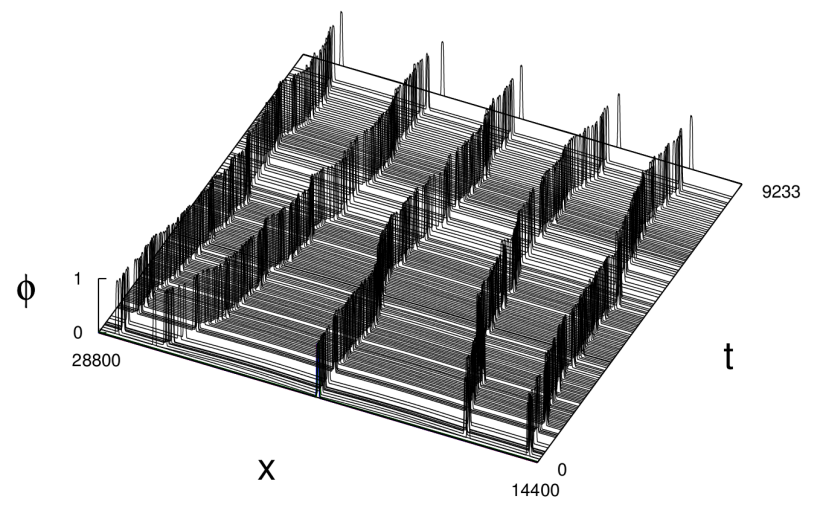

Fig. 12. Evolution from the initial condition $\phi_{0}^{\mathrm{u}}(x)$ in Eq. (14), when the system is submitted to noise with $\Gamma_{\mathrm{u}}$ and $\sigma=30$. Five localized patterns are elicited, out of which only the central one is "seeded". Parameters: $\varepsilon_{0}=14, \alpha=0.001$.
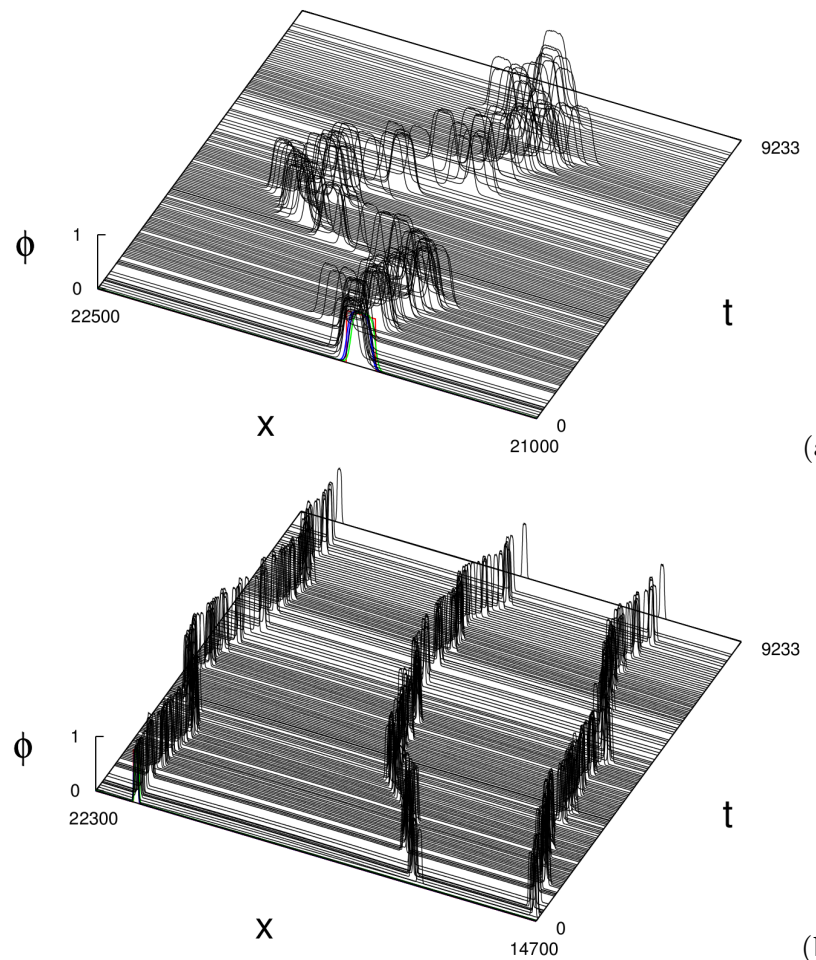

(a)

9233

Fig. 13. Zooms of Fig. 12: (a) focalizing on the "seeded" localized pattern, (b) including two spontaneous localized patterns. 
Figures 12 and 13 show the system's evolution for $\sigma=30$. In Fig. 12, five " $S^{+}$soliton" evolutions are recorded, out of which only the one displayed in Fig. 13 (a) is "seeded". Note how both shorter- and longer-timescale motions are enhanced because of the large noise intensity. Figure 13 (b) shows moreover the two spontaneous localized patterns at its right in Fig. 12. Even though large, the noise intensity is not yet large enough for the "soliton" density to significantly restrict their longer-timescale motion. A hint of their interaction is however provided by the fact that at longer times, the localized patterns tend to be equidistant.

Figures 14 and 15 show the system's evolution for $\sigma=40$. In Fig. 14, seven " $S^{+}$soliton" evolutions are recorded, out of which only the one displayed in Fig. 15 (a) is "seeded". Figure 15 (b) shows moreover the three spontaneous localized patterns at its right in Fig. 14. In comparison with the previous case, the longer-timescale motion looks more restricted, but the shorter-timescale one is enhanced. Finally, if $\sigma$ is still larger, " $S^{+}$solitons" are also destabilized.

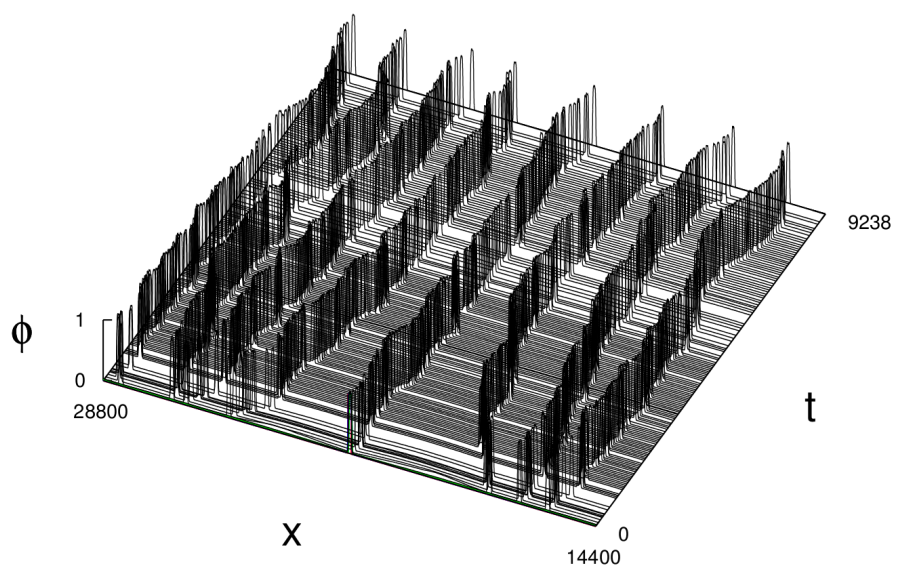

Fig. 14. Evolution of seven localized patterns, system triggered with $\phi_{0}^{\mathrm{u}}(x)$ and using $\Gamma_{\mathrm{u}}$, for $\sigma=40$ (here $\varepsilon_{0}=14$ and $\alpha=0.001$ ). Parameters: $\varepsilon_{0}=14$, $\alpha=0.001$. 

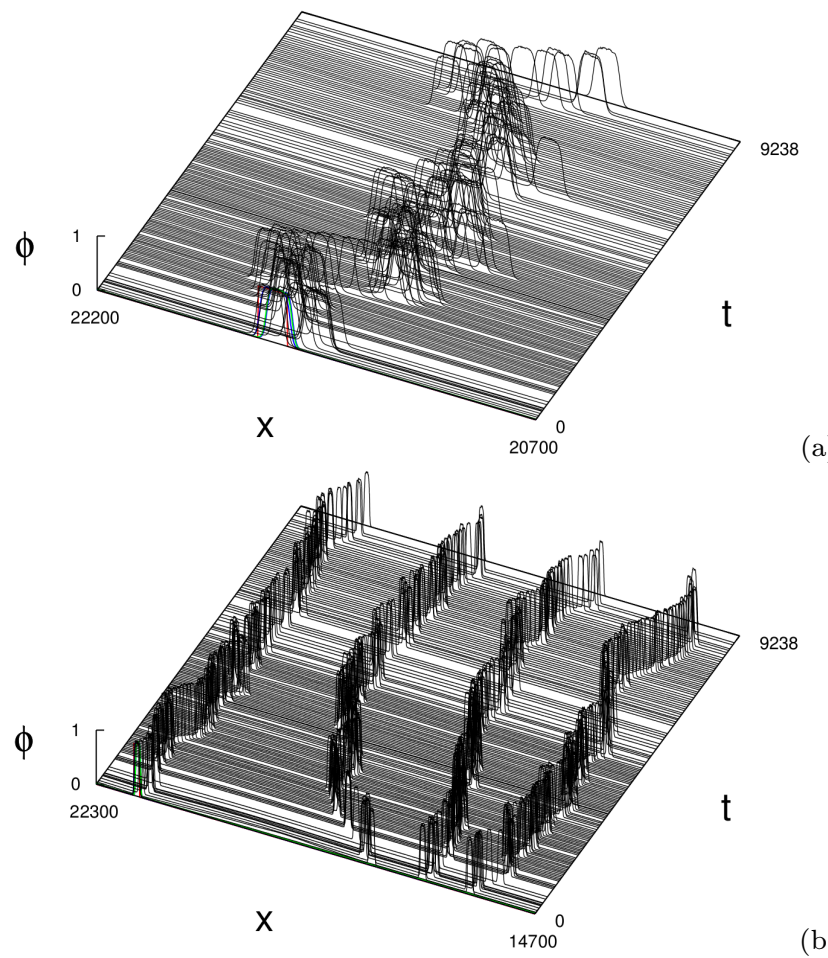

(a)

(b)

Fig. 15. Zooms of Fig. 14: (a) focalizing on the "seeded" localized pattern, (b) including three spontaneous localized patterns.

\section{Analysis and conclusions}

In this paper, we propose a method to stabilize localized patterns in a model describing a monolayer with attractive lateral interactions (ALI) between the adsorbates. Regardless of the parameter values, the dynamics governing such a system allows only propagating front-like structures. If one can however displace - by means of a multiplicative noise - only one of the two HS toward the "constructive region" in which the ALI can by themselves stabilize nanopatterns, a bistable pattern-HS situation is produced. In this way, pinning fronts - and therefore, localized patterns can be stabilized. The latter acquire moreover a stochastic motion. This motion can be conceptually decomposed into a (stochastic but nevertheless somewhat well-defined) path, and shorter-timescale fluctuations around it. In principle, the fluctuation of both components becomes wider as the noise intensity increases. However, since at larger noise intensities many spontaneous localized patterns are elicited, these interact and somewhat confine the longer-timescale component. 
Affecting only one HS requires the derivative of the noise's factor with respect to the field to be high enough, a requirement which is fulfilled by an exponential function but not by powers. The noise-induced "reaction term" drives the system toward a situation where noise effects are minimized. The new stationary HS results from the balance between the stochastic and deterministic forces.

The alluded multiplicative noise is of the kind that enables the "entropic mechanism" for noise-induced phase transitions [17, 18, 36, 37]. Following a series of articles discussing nanopattern stabilization by employing noises of this kind, we have reported recently similar results for a normal form [14]. The present application is not only more realistic, but also very much interesting, because the localized patterns emerge in an adsorbed metal surface and their size is of the order of the nanometers [19, 29, 38]. Clearly, although our proposal is at this stage theoretical in nature, we expect to rise the interest in the community about manipulating nanopattern production in adsorbed monolayers by means of external noise. At a macroscopic scale, this could be done in a photosensitive reaction by means of computer-controlled masks, implemented with the screen of a discarded but running laptop.

We acknowledge financial support from UNMdP (Projects EXA732/15 and EXA603/12) and from CONICET of Argentina.

\section{REFERENCES}

[1] M.G. Clerc, S. Coulibaly, D. Laroze, Phys. Rev. E 77, 056209 (2008).

[2] M.G. Clerc et al., Phys. Rev. E 84, 036205 (2011).

[3] M. Cross, H. Greenside, Pattern Formation and Dynamics in Nonequilibrium Systems, Cambridge University Press, Cambridge, UK, 2009.

[4] O. Descalzi et al., Editors, Localized States in Physics: Solitons and Uatterns, Springer, Berlin 2011.

[5] A.W. Liehr, Dissipative Solitons in Reaction Diffusion Systems: Mechanisms, Dynamics, Interaction, Springer, Berlin 2013.

[6] R. Carretero-González et al., Editors, Localized Excitations in Nonlinear Complex Systems: Current State of the Art and Future Perspectives, Springer, Berlin 2014.

[7] A. Gierer, H. Meinhardt Kybernetik 12, 30 (1972).

[8] M. Herschkowitz-Kaufman, G. Nicolis, J. Chem. Phys. 56, 1890 (1972).

[9] T.H. O'Dell, Editor, Magnetic Bubbles, Wiley, New York 1974.

[10] J. Wu, R. Keolian, I. Rudnick, Phys. Rev. Lett. 52, 1421 (1984).

[11] P. Coullet, C. Riera, C. Tresser, Phys. Rev. Lett 84, 3069 (2000). 
[12] P. Coullet, Int. J. Bif. Chaos Appl. Sci. Eng. 12, 2445 (2002).

[13] K. Kawasaki, T. Ohta, Physica A 116, 573 (1982).

[14] S.E. Mangioni, R.R. Deza, Phys. Rev. E 92, 032116 (2015).

[15] A. Mikhailov, G. Ertl, Chem. Phys. Lett. 238, 104 (1995).

[16] M. Hildebrand, A. Mikhailov, J. Phys. Chem. 100, 19089 (1996).

[17] M. Ibañes, J. García-Ojalvo, R. Toral, J.M. Sancho, Phys. Rev. Lett. 87, 020601 (2001).

[18] O. Carrillo et al., Phys. Rev. E 67, 046110 (2003).

[19] M. Hildebrand, A. Mikhailov, G. Ertl, Phys. Rev. E 58, 5483 (1998).

[20] S.E. Mangioni, Physica A 389, 1799 (2010).

[21] S.E. Mangioni, R. Deza, Phys. Rev. E 82, 042101 (2010).

[22] S.E. Mangioni, R.R. Deza, Physica A 391, 4191 (2012).

[23] S.B. Casal, H.S. Wio, S.E. Mangioni, Physica A 311, 443 (2002).

[24] S.E. Mangioni, H.S. Wio, Phys. Rev. E 71, 056203 (2005).

[25] J.A. Sierra, H.S. Wio, Centr. Eur. J. Phys. 10, 625 (2012).

[26] G.G. Izús, R.R. Deza, H.S. Wio, Eur. Phys. J. Spec. Top. 223, 167 (2014).

[27] V.O. Kharchenko, D.O. Kharchenko, Surface Sci. 637-638, 90 (2015).

[28] V.O. Kharchenko, D.O. Kharchenko, A.V. Dvornichenko, Physica A 444, 689 (2016).

[29] M. Hildebrand et al., Phys. Rev. Lett. 83, 1475 (1999).

[30] V.P. Zhdanov, Phys. Rev. E 55, 4826 (1997).

[31] J. Verdasca, G. Dewel, P. Borckmans, Phys. Rev. E 55, 4828 (1997).

[32] M.G. Clerc, E. Tirapegui, M. Trejo, Phys. Rev. Lett. 97, 176102 (2006).

[33] S.E. Mangioni, Eur. Phys. J. B 88, 53 (2015).

[34] S.E. Mangioni, Eur. Phys. J. B 88, 265 (2015).

[35] Y. Pomeau, Physica D 23, 3 (1986).

[36] J. Buceta, K. Lindenberg, Phys. Rev. E 69, 011102 (2004).

[37] J. Buceta, M. Ibañes, J.M. Sancho, K. Lindenberg, Phys. Rev. E 67, 021113 (2003).

[38] M. Hildebrand, Chaos 12, 144 (2002). 